\title{
Pandemias, COVID-19 y Salud Mental: ¿Qué Sabemos Actualmente?
}

\author{
Pandemics, COVID-19 and Mental Health: What Do We Know Today?
}

\author{
Alfonso Martínez-Taboas ${ }^{{ }^{*}}$ \\ 1 Universidad Interamericana de Puerto Rico, Puerto Rico. (D) https://orcid.org/0000-0001-8331-0423 \\ * Correspondencia: amtaboas@coqui.net
}

Recibido: 8 junio 2020 | Aceptado: 29 junio 2020 | Publicado: 15 julio 2020

WWW.REVISTACARIBENADEPSICOLOGIA.COM

\begin{abstract}
Citar como:
Martínez-Taboas, A. (2020). Pandemias, COVID-19 y Salud Mental: ¿Qué Sabemos Actualmente? Revista Caribeña de Psicología, 4(2), 143-152. https://doi.org/10.37226/rcp.v4i2.4907
\end{abstract}

\begin{abstract}
RESUMEN
Las pandemias tienen la característica nefasta de crear un disloque masivo y generalizado en múltiples niveles, tanto personales como sociales. Las pandemias suelen relacionarse con confusión, temores, incertidumbre y la probabilidad de muertes en seres queridos. Asimismo, se relacionan con innumerables estresores sociales tales como hacer ajustes en las rutinas, separación de amigos y familiares, pérdida de empleos y aislamiento social. El surgimiento del COVID-19 hacia finales del 2019 ha traído consigo muchos de esos estresores, pero en una escala que no veíamos desde la pandemia del 1918, la cual se calcula que contaminó a casi una tercera parte de la humanidad. En este artículo se presentan datos recientes internacionales, que han examinado cómo el COVID-19 ha impactado la salud mental de innumerables personas. Se desprende de esta revisión que hay datos contundentes de que algunos trastornos psiquiátricos han aumentado notablemente, en especial la ansiedad, depresión, insomnio, y temores generales. Esto se ha encontrado en niños, adolescentes y adultos. La tasa de trastornos mentales es más alta aún en personas contagiadas y en los trabajadores de salud que se enfrentan día a día a tratar personas con COVID-19. Asimismo, se han identificado ciertos factores de riesgo, tales como tener puntuaciones altas en ansiedad rasgo, ser mujer, y haber estado cerca de personas contagiadas. Finalizamos haciendo un acercamiento conceptual y teórico hacia el tema y esbozando un resumen de cómo ayudar a minimizar el trastoque psicosocial de esta pandemia.
\end{abstract}

Palabras Claves: pandemia; COVID-19; ansiedad; depresión; salud mental; aislamiento social

\section{ABSTRACT}

Pandemics are associated with a score of social and medical stressors that create severe disruptions at multiple levels. Pandemics are related to confusion, fears, uncertainty and the probable deaths of friends and loved ones. Also, pandemics are associated with a wide variety of social stressors such as economic and job loss, social isolation, the breakdown of healthcare systems and other pleasant routines. The manifestation of COVID-19 on December 2019 has created innumerable social and personal stressors that incidentally are similar to the pandemic of 1918 that contaminated nearly a third of the world population. In this article, I present recent empirical international studies that have examined the impact of COVID-19 on the mental health of innumerable people's around the world. The data are consistent: some psychiatric disorders have shown a dramatic increase including anxiety, 
depression, insomnia and general fearfulness. This finding is generalizable in children, adolescents and adults. Medical professionals that work with COVID-19 patients show a dramatic increase in those psychiatric disorders. On the other hand, the literature has identified some populations at risk of developing a psychiatric disorders, such as previous trait anxiety, being a woman, and having been exposed to a person with COVID-19. I conclude with a conceptual and theoretical approach resuming different multilevel tactics that could minimize the mental health impact of the pandemic.

Keywords: pandemic; COVID-19; anxiety; depression; mental health; social isolation

\section{INTRODUCCIÓN}

Vamos a empezar con una premisa inequívoca: la humanidad a través de milenios se ha tenido que enfrentar a innumerables desastres naturales, desastres producidos por los propios seres humanos, innumerables plagas y enfermedades. En ocasiones la miseria y destrucción es impresionante. Por ejemplo, durante el gran terremoto del 1755 en Lisboa, con una intensidad calculada de 9.0, unas 70,000 personas murieron en pocos minutos producto del efecto del terremoto en más de 12,000 edificios y viviendas y un tsunami calculado en 10 metros que dejó a Lisboa en la pura destrucción (Molesky, 2015). Asimismo, lo que se conoce como la plaga de la peste negra, la cual se manifestó principalmente entre el 1348-1361 mató aproximadamente a un cuarto de toda la población de Europa y Asia, calculándose en unos 24 millones de muertes (Cartwright \& Biddiss, 2004). Más recientemente, la llamada gripe española, que duró del 1918 hasta el 1920, ocasionó la muerte en unas 50 millones de personas y llegó a contagiar a un tercio de toda la humanidad. De esos 50 millones de personas que fallecieron, unas 675,000 ocurrieron en los EEUU (Spinney, 2017). En este artículo voy a ofrecer una revisión de literatura, de corte internacional, sobre lo que sabemos de la pandemia del COVID-19 y su relación con trastornos emocionales y el bienestar y felicidad de las personas.

\section{COVID-19: ¿DE QUÉ SE TRATA?}

El primer caso reportado de una neumonía atípica se reportó en Wuhan, Provincia de Hubei de la República Popular China el 30 de diciembre de 2019. Ya para el 7 de enero 2020 se identificó el síndrome severo respiratorio agudo coronavirus, conocido en la literatura como SARS-CoV-2. Al momento en que escribo (24 de junio), según el Johns Hopkins Coronavirus Resource Center, el COVID-19 ha sido identificado en 181 países/regiones, con 9,295,365 millones de casos confirmados y ha causado 478,289 muertes. Los datos iniciales provenientes de China indicaban que el $80 \%$ presenta síntomas leves, el $15 \%$ hospitalización y un $5 \%$ requiere cuidado intensivo. Pero un estudio reciente con una muestra de 72,314 individuos infectados en China, reveló que el $14 \%$ mostró enfermedades serias y una fatalidad de $2.3 \%$ (Wu \& McGoogan, 2020). Factores de riesgo para mortandad son: tener más de 60 años de edad, y tener comorbilidades médicas tales como complicaciones cardiovasculares, diabetes, cáncer y problemas respiratorios. El promedio de incubación es de 3 a 6 días y luego de esto, en casos que se complican, la hospitalización se requiere luego de unos 7 días desde el comienzo de la enfermedad. La complicación más común es el síndrome agudo de dificultad respiratoria (Cevik et al., 2020). En los EEUU el COVID-19 ha sido más mortal y virulento con 2,348, 956 casos confirmados y 121,279 muertes. O sea, los EEUU recogen el $25 \%$ de todas las muertes mundiales, a pesar de que la población de EEUU es de un $5 \%$ a nivel mundial.

\section{IMPACTO EN LA SALUD MENTAL}

A pesar de que el COVID-19 es de reciente aparición, en los pasados cuatro meses se han publicado estudios bastante rigurosos, algunos de corte epidemiológico, sobre cómo las personas han reaccionado a nivel emocional y conductual, a esta pandemia. Como veremos, el efecto ha sido muy marcado y notable en una cuantiosa parte de la población general y en personas que, por la naturaleza de su trabajo, se exponen al virus. Para entender las razones y causas de este impacto negativo en la salud mental, tenemos que tener en cuenta los siguientes dos factores:

Primero, el COVID-19 ha tenido un efecto negativo enorme en el número de personas que han perdido su empleo. De acuerdo a datos del Pew Research Center, de 6.2 millones de desempleados en febrero, aumentó a 20.5 millones en mayo (Koshhar, 2020). De 
hecho, el número de desempleados es mucho mayor a la llamada Gran Recesión del 2007 al 2009. En el 2020 el desempleo en los EEUU subió de un $3.8 \%$ en febrero a un $14.4 \%$ en abril. En la Gran Recesión el pico mayor fue de un 10.6\% (Koshhar, 2020). Este dato es muy importante porque hay una abundante literatura que apunta a una fuerte relación entre estar desempleado y declives en salud mental. Por ejemplo, recientemente Forber y Krueger (2019) realizaron un estudio longitudinal del 2003 al 2013 sobre el impacto de la Gran Depresión en personas que perdieron su empleo. Los hallazgos revelaron que dichas personas informaron un incremento en niveles de ansiedad generalizada, pánico, depresión y uso problemático de substancias, aun cuando habían pasado 34 años luego de que terminara la recesión.

Crayne (2020) ha argumentado que el problema del desempleo no sólo es la potencial pérdida financiera. También mucha gente encuentra en su trabajo un motivo de sentido de vida, compañerismo, bienestar, y de expresión de valores personales. Crayne (2020) presenta datos de que para algunas personas perder su empleo es incluso traumático y tiene el potencial de generalizar su malestar con su entorno familiar. Además, menciona que hay datos que indican que la búsqueda subsecuente de un nuevo empleo puede ser fuente de frustraciones y ansiedades.

En segundo lugar, está el impacto negativo del distanciamiento social y de la cuarentena social. En EEUU el estado de California fue el primero en implementar una cuarentena social, cerrándose escuelas, universidades, centros comerciales, iglesias, parques de entretenimiento, restaurantes y barras. Por ejemplo, Tull et al. (2020), utilizaron una muestra nacional en los EEUU con 500 personas. Los resultados revelaron que las personas que cumplían con no salir de sus casas se asociaban a presentar más ansiedad de salud, más preocupaciones financieras y sentirse muy solos. Es muy probable que, en ausencia de las relaciones interpersonales significativas, los trastornos de ansiedad y depresión surjan o empeoren. Asimismo, la cuarentena dificulta para muchas personas la disponibilidad de intervenciones psicoterapéuticas y de asesoramiento. Estos hallazgos resaltan la importancia de la conexión social para minimizar los efectos negativos del aislamiento del COVID-19.

\section{Salud Mental en General}

Algunos estudios han utilizado escalas globales que miden psicopatología. Un buen ejemplo, es el estudio de Tian et al. (2020) realizado en China. Para este estudio participaron 1,060 adultos entre el 31 de enero y el 2 de febrero 2020. Los resultados revelaron unas puntuaciones altas de malestar psicológico en el Symptom Checklist-90. Para sacar estas conclusiones los autores usaron las normas nacionales de China en el SCL-90. Así, por ejemplo, más del 70\% de los participantes obtuvieron puntuaciones clínicas altas (moderadas a severas) en ansiedad, obsesiones, y sensibilidad interpersonal. Personas en riesgo eran las que tenían más de 50 años, eran divorciados o viudos, tenían poca educación, y trabajadores de la agricultura.

\section{Ansiedad, Estrés y Depresión}

Un hallazgo consistente en la literatura es que los síntomas o trastornos de ansiedad y depresión son los que más se reportan en estudios que indagan la salud mental de personas en la pandemia. Mucha gente parece estar desesperada y muy angustiada por la situación. Por ejemplo, las líneas de emergencia federales que registran personas con angustias emocionales, registraron un incremento del 1,000\% en abril 2020, comparado con abril 2019. También la compañía Talkspace, que se dedica a dar servicios en línea por crisis emocionales, reportó un incremento del 65\% prácticamente todas relacionadas con preocupaciones con el COVID-19 (Andrade, 2020).

Algunos ejemplos recientes ejemplificarán el impacto del COVID-19 en estos parámetros. Qui et al. (2020) en un estudio con 52,230 participante de China, encontró que el $35 \%$ de la población estaba experimentando un estrés fuerte, principalmente las mujeres y personas de mayor edad. Por su parte, Wang et al. (2020) con 1,210 participantes de China encontró que un $24.5 \%$ presentaba síntomas mínimos, un $21.7 \%$ síntomas moderados y un $53.8 \%$ síntomas severos en una escala que medía estrés general. En este estudio, nuevamente las mujeres registraron mayor estrés.

Huang y Hao (2020) en un estudio nacional en China, con 7, 236 participantes, encontró un número alto de personas con síntomas significativos que afectaban su salud mental. Por ejemplo, el 35.1\% cumplía con criterios de ansiedad generalizada, un $20.1 \%$ con 
depresión y un $18.2 \%$ con insomnio. Estos porcentajes son muchos más altos que estudios de prevalencia previos realizados en China. Las personas en riesgo eran las que invertían más de 3 horas diarias pensando activamente en la pandemia.

Por su parte, Gao et al. (2020) utilizaron datos de un estudio con 4,872 participantes en 31 provincias de China y encontraron niveles altos de depresión y ansiedad. Por ejemplo, un 48.3\% reportaron síntomas notables de depresión, un $22.6 \%$ ansiedad y un $19.4 \%$ una mezcla de ansiedad y depresión. Los autores encontraron que los participantes que pasaban muchas horas en plataformas sociales leyendo del COVID 19, presentaban más síntomas severos.

Recientemente están apareciendo estudios que no se limitan a China o EEUU. Por ejemplo, Roy, et al., (2020), realizaron un estudio en la India con 662 personas que respondieron a una encuesta en línea. Los resultados más interesantes es que el $80 \%$ informó sentir mucha ansiedad y preocupación por el virus. Un $72 \%$ en la última semana se sintió muy preocupado con adquirir el COVID-19 o alguno de sus familiares cercanos. Un $12 \%$ no podían dormir bien, y un $41 \%$ admitió sentir miedo. Un $83.5 \%$ de los participantes dijeron que desearon hablar con algún experto de salud mental sobre su situación, pero no tenían los recursos para hacerlo.

Por su parte, Jungmann y Withoff (2020) realizaron un estudio en Alemania con una muestra de 1,615 personas en línea. Su estudio se enfocó en niveles de ansiedad en la población. Los autores encontraron un incremento notable en los niveles de ansiedad desde antes de la pandemia, hasta el mes de abril. Por ejemplo, en la escala de ansiedad que utilizaron, en el mes de diciembre 2019 obtuvieron una puntuación baja de 8.30; en enero subió a 19.00 y en abril a 47.18. Según estos datos, el 25\% de la población indicó sentir síntomas de ansiedad moderados/severos y el otro $25 \%$ síntomas extremos de ansiedad. En este estudio, el tener rasgos de ansiedad (trait anxiety) altos antes de la pandemia, fue un predictor de tener problemas con manejar la ansiedad. En este estudio las mujeres nuevamente reportaron un nivel bastante más alto de ansiedad que los hombres.

En un estudio original y novedoso, Li et al. (2020) usaron los datos en línea de usuarios de un lugar cibernético que se llama Weibo. En esta plataforma los usuarios suelen discutir sus asuntos de interés general. Los autores obtuvieron datos de 17,865 usuarios que escribieron del 13 de enero hasta el 26 de enero 2020. Utilizando indicadores linguísticos, los autores encontraron que según iban aumentando los días, aumentaban las palabras relacionadas con las siguientes categorías: ansiedad, depresión, indignación, y emociones negativas. Asimismo, hubo un decremento en palabras de felicidad y compartir con amistades.

En un estudio en Irán, con una población de 10,754 personas, Moghanibashi (2020) se enfocó en la ansiedad reportada. Del estudio se desprende que un 51\% reportó algún nivel de ansiedad y un 19\% ansiedad extrema. Las mujeres y las personas que tenían algún familiar con COVID-19 reportaron puntuaciones más altas de ansiedad. En otro estudio en Turquía, Ozdin y Ozdin (2020) reportan datos de un estudio en línea entre la población general. Participaron 343 personas. Los hallazgos más importantes son los siguientes: Un $45.1 \%$ reportó síntomas consistentes con ansiedad; un $23.6 \%$ con síntomas marcados de depresión. Al utilizar un análisis de regresión, se encontró que ser mujer, vivir en áreas rurales y tener un historial psiquiátrico previo, fueron factores de riesgo.

Se pueden citar a nivel individual otros estudios, pero prefiero utilizar una revisión de literatura sistemática que realizaron Vindegaard y Benros (2020). Los autores identificaron 43 estudios publicados sobre salud mental y COVID-19. Los tres hallazgos más interesantes son los siguientes:

1. De 714 pacientes con COVID-19 que estaban hospitalizados, el 96.2\% informó síntomas de estrés postraumátco. Esto implica que tener $\mathrm{CO}$ VID-19 es una experiencia muy traumática y amenazante, probablemente por temer a perder la vida.

2. Los pacientes que ya de antemano tenían trastornos psiquiátricos, mostraron una exacerbación de sus síntomas entre un $30 \%$ a un $50 \%$ de los casos.

3. Los factores de riesgo que predisponían a las personas a presentar complicaciones emocionales eran los siguientes: ser mujer, una vida de mucho estrés, eventos traumáticos anteriores, tener poco conocimiento de la pandemia, y tener poco apoyo social. Notemos como en casi todos 
los estudios se repite de manera consistente la variable de ser mujer como factor de riesgo.

\section{Salud Mental en Trabajadores de la Salud}

Desde muy temprano en la pandemia, quedó claro que las personas que trabajan en el campo de la salud, y muy en especial los que laboran con la atención de enfermos por COVID-19 en hospitales, presentaban unos riesgos considerables a su salud física como mental. Los siguientes estudios fundamentan esta noción. Lai et al. (2020) estudiaron la salud mental de 1,257 enfermeras y médicos en 34 diversos hospitales en China. Los niveles de reacciones emocionales adversas fueron muy altos. Por ejemplo, el $71 \%$ dijo sentirse angustiado, un 50\% con síntomas de depresión, un $45 \%$ con síntomas de ansiedad y un $34 \%$ con insomnio. Las enfermeras, las mujeres y los profesionales de salud de primera línea, informaron un mayor número de síntomas. Recordemos que los trabajadores de primera línea tienen un contacto más directo con el paciente, haciendo el diagnóstico, tratamiento y cuido.

El riesgo de contagio y muerte es un fenómeno real en los profesionales de la salud que trabajan directamente con pacientes con COVID 19. Por ejemplo, Girolamo et al. (2020) documentan la experiencia en Italia. Sus datos demuestran que, en Italia, para abril 16 de 2020, 16, 991 personal de la salud había sido infectado, y 127 médicos habían muerto como consecuencia del contagio. Por su parte, Rossi et al. (2020), en un estudio en Italia con 1,379 personas relacionadas a trabajar con la salud, un $49 \%$ endosó síntomas agudos de estrés post-traumático, un $25 \%$ síntomas de depresión, un $8 \%$ insomnio y un $22 \%$ percibían mucho estrés. Los síntomas de depresión y de estrés post-traumático eran más comunes en mujeres. En otro estudio llevado a cabo por Zhu et al. (2020) en Gansu, China, se evaluó la salud mental de 86 enfermeras y 79 doctores. Los resultados indicaron que los doctores informaban un $45.6 \%$ de depresión y un $11.4 \%$ de ansiedad. Las enfermeras informaban un $43 \%$ de depresión y un $27.9 \%$ de ansiedad.

\section{¿Qué Sabemos Sobre Menores de Edad y Salud Mental?}

La mayoría de la literatura de corte nacional y epidemiológico se ha realizado con adultos. Por lo tanto, sólo hay unos pocos estudios sobre el impacto del
COVID-19 en los niños. Ya de entrada sabemos que esta pandemia ha creado un desbalance en los estilos de vida de los niños: dejar de jugar deportes, no asistir a la escuela, miedos, aislamiento social y miedo a la muerte. En un estudio informado por Jiao et al. (2020), llevado a cabo en la provincia de Shaanxi (China), con 168 niñas y 142 niños de 3-18 años, se reportaron niveles altos de conductas de apego excesivo, irritabilidad, miedos y distracción.

Para tomar un estudio adicional ilustrativo, Zhou et al. (2020) realizaron un estudio de corte epidemiológico entre estudiantes chinos de 12-18 años ( $n=$ $8,079)$. El hallazgo más relevante fue que un porcentaje alto y significativo informó angustias emocionales en esta población. Por ejemplo, el $43.7 \%$ reportó síntomas significativos de depresión, otro $37.4 \%$ síntomas marcados de ansiedad y un 31.3\% reportó una combinación de depresión y ansiedad. En otros análisis realizados, encontraron que el principal factor de riesgo era ser mujer, seguido de estar ubicado en el último grado de estudio.

\section{BIENESTAR PSICOLÓGICO Y GENERAL}

Aunque la literatura se ha enfocado en temas de salud mental y trastornos psiquiátricos, hay unos pocos estudios que le dan una mirada a aspectos más amplios como satisfacción de vida, bienestar y problemas de tipo social. El estudio de Yang y Ma (2020) es un buen ejemplo. Estos autores informan datos de un estudio con 14,131 residentes de 34 provincias en China. Lo interesante de este estudio es que los participantes llenaron las mismas escalas antes del anuncio del COVID-19 (diciembre 2019) y a mitad de febrero 2020. Los resultados son dramáticos. En la escala que mide bienestar emocional, $74 \%$ de los participantes informaron un declive sostenido. Los autores identificaron ciertos factores que se relacionaron con dicho declinamiento, entre ellos: a) las personas que residían en Hubei, que fue el epicentro del brote, reportaron un declive mayor que en cualquiera de las otras provincias; b) las personas mayores de 60 años también reportaron menor bienestar, probablemente porque ya para febrero se sabía que el COVID-19 es más virulento y peligroso en personas mayores; c) las personas casadas también sufrieron un declive mayor, quizás debido a que estar confinado por largo tiempo con otra persona, trae a flote problemas de la relación y las angustias que esto trae. Por otro lado, 
en una serie de regresiones estadísticas, los autores encuentran evidencia de dos variables protectoras del bienestar. La primera se relaciona con la percepción de que la persona está bien informada del COVID-19. La segunda es que la persona piensa que tiene cierto control de la situación circundante. Los autores concluyen diciendo que "la percepción del participante de que tiene conocimiento sobre el coronavirus fue asociada con un nivel más alto de control, lo que a su vez protegió su bienestar emocional durante el brote" (p. 5).

En otro estudio reciente, que proviene de la NORC de la Universidad de Chicago (2020), los investigadores tomaron una muestra representativa de los 50 estados de la nación estadounidense. En total, participaron 2,279 personas que incluyeron las fechas de 21 de mayo al 29 de mayo 2020. Para comparar los resultados obtenidos, usaron un grupo de comparación de personas encuestadas en el 2018 para los estudios del General Social Survey que se realizan anualmente en los EEUU. Algunos de los datos más sobresalientes son los siguientes: a) en el 2018 el 31\% de los norteamericanos se describían como muy felices. Pero en mayo 2020 esto bajó a un 14\%, o sea, una reducción notable de $17 \%$; b) en el 2018 un $23 \%$ indicaba sentirse solo, pero en el 2020 esto aumentó a un 50\%. Este estudio también dividió a los participantes en dos grupos. Por un lado, aquellas personas que en el último mes estuvieron cerca de una persona que se le diagnosticó el COVID-19 (13\%) y aquellas personas que negaban esta experiencia (87\%). Los resultados fueron reveladores. En todas las preguntas realizadas, las personas que habían sido expuestas reportaban mayores de los siguientes problemas (Tabla 1):

Tabla 1

Personas expuestas o no expuestas al COVID-19.

\begin{tabular}{lcc}
\hline \multicolumn{1}{c}{ Síntomas } & $\begin{array}{c}\text { No estuve cerca } \\
\text { de alguien con } \\
\text { COVID }\end{array}$ & $\begin{array}{c}\text { Estuve cerca de } \\
\text { alguien con } \\
\text { COVID }\end{array}$ \\
\hline Tenso & 43 & 60 \\
Cansado & 45 & 55 \\
Dolor de cabeza & 34 & 52 \\
Llantos & 32 & 48 \\
Malestar estómago & 26 & 47 \\
Irritado & 24 & 44 \\
Sin apetito & 21 & 37 \\
Taquicardia & 17 & 29 \\
Sudoroso & 8 & 24 \\
Fumar mucho & 13 & 25 \\
Insomnio & 48 & 61 \\
\hline
\end{tabular}

Estos datos revelan que la pandemia del COVID19 no solo ha aumentado la incidencia de trastornos psiquiátricos, sino que también ha trastocado el bienestar general de las personas, su felicidad, y un aumento notable en malestares somáticos y dificultades para dormir.

\section{VARIABLES RACIALES Y ÉTNICAS}

Diversos estudios de corte epidemiológico han documentado, desde hace décadas, disparidades en la salud general de la población estadounidense y barreras para una mejor calidad de vida (Rumbold \& Dickson, 2012). Con esto nos referimos a los determinantes sociales de la salud. No nos debe sorprender que el COVID-19 haya puesto en una vulnerabilidad excesiva a personas de raza negra o de diversas etnias Latinas. Datos recientes apoyan esta idea. Por ejemplo:

1. La pandemia ha afectado adversamente mucho más a personas afroamericanas. Algunos ejemplos: En Luisiana las personas de raza negra comprenden un $32 \%$ de la población, pero aportan un $70 \%$ de las muertes. En Illinois personas de raza negra comprenden un $39 \%$ de la población local, pero el 56\% de las muertes son de personas negras. En Michigan las personas de raza negra componen el 14\% de la población general, pero el $41 \%$ de las muertes por COVID-19 se dan en personas negras (Artiga et al., 2020).

2. Hooper et al. (2020) tienen datos precisos calculados por 100,000 habitantes. Por ejemplo, el nivel de mortalidad en Nueva York (al 7 de mayo) es de 187/100,000 en latinos, 184/100,000 en afroamericanos y 93/100,000 en blancos. En Chicago, es de 73/100,000 en afroamericanos, 36/100,000 en latinos y 22/100,000 en blancos.

Las razones para esto pueden ser las siguientes: las minorías presentan más complicaciones de salud (diabetes, enfermedades cardiovasculares, obesidad y asma) y más comorbilidades que personas de raza blanca. Tienen más dificultades en obtener pruebas y tratamiento, debido a que muchos no están asegurados con planes médicos. Las minorías raciales reportan más barreras para obtener servicios médicos de calidad. Los afroamericanos tienden a vivir en comunidades y localizaciones con alta densidad pobla- 
cional que los ponen en riesgo de contagiarse del virus (Artiga et al., 2020).

\section{OTRAS VARIABLES RELACIONADAS A UN MENOSCABO DE SALUD MENTAL}

De la revisión de literatura reciente que realizaron Hossain et al. (2020) se desprenden las siguientes variables de alto riesgo:

1. Hay una tendencia en la literatura de que las personas jóvenes (< 40 años) padecen más de depresión y ansiedad ante la pandemia. Es posible que esto se deba a que están más conectados a las redes sociales leyendo o conversando del tema.

2. Las mujeres tienen de tres a nueve veces más probabilidad de presentar ansiedad y depresión en la pandemia. Esto ha sido replicado en innumerables estudios.

3. Varios estudios han encontrado una relación inversa entre educación y angustias psiquiátricas. Por lo tanto, tener mayor educación parece ser un factor protector. Quizás esto se deba a que la persona con mayor educación puede entender mejor cómo protegerse y balancear la información que lee y a su vez ser más escéptico a las innumerables teorías de conspiración.

4. Los factores económicos también constituyen otro factor de riesgo. Estudios en varios países del mundo indican que problemas económicos serios, perder el hogar y estar desempleado son factores de riesgo.

5. El lugar de residencia y contacto próximo con personas con COVID-19 es un factor de riesgo fuerte para muchas personas. $\mathrm{O}$ sea, personas que viven en ciudades o comunidades en donde hay tasas altas de contagios y mortandan tienden a reportar mucha más ansiedad y depresión.

6. Presentar un historial previo de abuso de sustancias, depresión y ansiedad pone a más personas en riesgo de desarrollar complicaciones emocionales, incluyendo doctores y enfermeras.
7. Varios estudios llevados a cabo en China han encontrado, de manera persistente, que estar oyendo noticias o exponerse a las redes sociales por más de 2 horas diarias para enterarse de asuntos del COVID-19, trae consigo preocupaciones, ansiedad y depresión.

\section{HACIENDO SENTIDO DEL PANORAMA}

Numerosos estudios a nivel internacional no dejan lugar a dudas de que la pandemia del COVID-19 ha dejado secuelas adversas psicológicas en un número creciente de ciudadanos. En particular, los síntomas de ansiedad, depresión, estrés e insomnio han aumentado significativamente y a veces llegan casi a un $50 \%$ de la población. Las personas que trabajan de cerca con personas contagiadas reportan síntomas más severos y marcados. Esto se ha reportado en Irán, EEUU, China, Alemania, Turquía, Italia y otros países donde se han hecho estudios poblacionales.

Un hallazgo que se repite a lo largo de los datos presentados es que las mujeres reportan más ansiedad y depresión que los hombres. ¿Debe sorprendernos esto? Entiendo que no. Desde hace décadas hay estudios que documentan, en docenas de diferentes países, que las mujeres muestran más síntomas de internalización ante situaciones estresantes (Freeman \& Freeman, 2013). Recordemos que síntomas de internalización son ansiedad, depresión, somatización y disociación. Los hombres, por su parte, muestran más síntomas de externalización, tales como uso de drogas, delincuencia, y conducta anti-social (Piquero et al., 2012)

Un estudio que acaba de salir de la Fundación Kayser tira una luz considerable sobre el asunto (Connoll, 2020). En este estudio se utilizó una metodología de encuesta por teléfono con 1,226 adultos durante el mes de marzo de 2020. Veamos los resultados: $49 \%$ de las mujeres y $40 \%$ de los hombres dijeron su vida había cambiado mucho por la pandemia; el 53\% de las mujeres dijo que su salud mental se había impactado negativamente por la pandemia, comparado con un $37 \%$ de los hombres; el $57 \%$ de las madres indicó lo mismo, frente a un $32 \%$ de los padres. Otros resultados son similares.

¿A qué se puede deber esto? Probablemente a la multiplicidad de roles y responsabilidades de las mujeres. En un estudio clásico de tipo longitudinal, 
Nolen-Hoeksema et al. (1999) le dieron un seguimiento de un año a 1,100 personas del estado de California que tenían de 25 a 75 años Tal y como se esperaba, las mujeres fueron abatidas más por la depresión que los hombres. Lo que este estudio aporta es que se dirige hacia la explicación de esto. Citamos a las autoras: "Los resultados de este estudio sugieren que las mujeres cargan con una tríada de vulnerabilidades hacia síntomas depresivos cuando se le compara con los hombres: más estrés crónico; una tendencia mayor a rumiar cuando están en estrés; y un sentido menor de tener dominio sobre sus vidas" (p. 1071).

Por ejemplo, en el estudio de Kayser (2020), las madres dijeron que durante la pandemia tenían el papel de maestras, cocinar, llevar las riendas de la casa, tener un empleo, y ser el apoyo principal en momentos de estrés. Todo esto ocurre mientras se les limitan sus oportunidades de esparcimiento, hacer ejercicios y conectarse en salidas con amigas o colegas del trabajo. No podemos negar también que hay estudios de laboratorio con medidas de neuro-imágenes que han indicado que cuando en un laboratorio se inducen escenas de miedo y estrés, los neuro-circuitos relacionados a estructuras de ansiedad de las mujeres tienden a activarse más que los hombres (Seo et al., 2017).

Hay muchos otros temas que se podrían desarrollar sobre el impacto del COVID-19 pero que en este breve espacio no tenemos para abordar. Por ejemplo: ¿Cómo es la resiliencia de todas esas personas que siguen funcionando de manera normal u óptima? ¿Estos síntomas de ansiedad y depresión cederán con el tiempo, o al contrario, seguirán afectando a un grupo grande de la población? ¿Los servicios de telemedicina y servicios de psicoterapia en línea serán igual de efectivos y traerán niveles altos de satisfacción en los pacientes? ¿Cuál debe ser el papel de los psicólogos en la respuesta macro-social a esta pandemia? ¿Habrá una incidencia más alta de lutos complicados a la luz de que muchas personas han muerto aisladas y sin tener sus seres queridos a su lado? ¿Cómo serán las luchas espirituales de los creyentes ante una pandemia que ha trastocado para mal la vida de tantas millones de personas? Estas preguntas, y muchas otras, ameritan de por sí otros ensayos adicionales.

No quiero terminar este ensayo sin al menos presentar el modelo de Bao et al. (2020) el cual fue propuesto por eruditos de la Universidad de Pekín para orientar en la toma de decisiones ante esta pandemia. Este modelo, que consta de 5 niveles (véase Tabla 2), es uno que impacta desde actitudes, creencias y conductas, conducentes a un mejor enfrentamiento ante una pandemia que estará con nosotros por años.

\section{Tabla 2}

Modelo de Toma de Decisiones ante la Pandemia (Bao et al., 2020).

\begin{tabular}{|c|c|c|}
\hline Nivel & Nombre & Actividad \\
\hline 1 & $\begin{array}{l}\text { Evaluar la } \\
\text { información que se } \\
\text { trasmite a la } \\
\text { población }\end{array}$ & $\begin{array}{l}\text { Que la información sea } \\
\text { confiable, basada en evidencia } \\
\text { y entendible por el público } \\
\text { general. }\end{array}$ \\
\hline 2 & $\begin{array}{l}\text { Mantener activos y } \\
\text { funcionales los } \\
\text { apoyos sociales }\end{array}$ & $\begin{array}{l}\text { Los apoyos vitales como } \\
\text { familia, amistades, } \\
\text { compañeros de trabajo, } \\
\text { miembros de iglesias deben de } \\
\text { continuar activamente } \\
\text { comunicándose por diversos } \\
\text { medios. }\end{array}$ \\
\hline 3 & $\begin{array}{l}\text { Reducir posibles } \\
\text { estigmas alrededor } \\
\text { de estereotipos de } \\
\text { la pandemia. }\end{array}$ & $\begin{array}{l}\text { Combatir mitos, falacias y } \\
\text { teorías de conspiración que } \\
\text { muchas veces crean un } \\
\text { ambiente de suspicacia y } \\
\text { culpas. }\end{array}$ \\
\hline 4 & $\begin{array}{l}\text { Tener una vida } \\
\text { segura y lo más } \\
\text { normal posible }\end{array}$ & $\begin{array}{l}\text { Énfasis en la protección } \\
\text { personal y de la familia, } \\
\text { mientras se continúa una vida } \\
\text { lo más cercana a la } \\
\text { normalidad y manteniendo } \\
\text { estilos nuevos de vínculos y } \\
\text { trabajos. }\end{array}$ \\
\hline 5 & $\begin{array}{l}\text { Utilizar los } \\
\text { servicios privados } \\
\text { o gubernamentales } \\
\text { que permitan la } \\
\text { recuperación } \\
\text { emocional de sus } \\
\text { ciudadanos }\end{array}$ & $\begin{array}{l}\text { Orientar a la ciudadanía en las } \\
\text { diversas opciones que existen } \\
\text { para manejar y superar sus } \\
\text { temores, ansiedades y tristeza. } \\
\text { Aquí se le da énfasis a } \\
\text { servicios psico-sociales y de } \\
\text { psicoterapia a distancia. }\end{array}$ \\
\hline
\end{tabular}

\section{CONCLUSIÓN}

En conclusión, las pandemias suelen traer consigo la necesidad imperiosa de lidiar con sus manifestaciones y secuelas, las cuales suelen estar inmersas en incertidumbre y ponen de manifiesto la fragilidad de nuestro entorno y la vida. No es de sorprendernos que, ante este escenario, la salud mental de una parte sustancial de la población sea impactada de manera negativa. La literatura apoya esta noción. Nos toca, pues, a cada uno de nosotros, ser responsables con nuestro autocuidado, tener una vida con propósito y 
sentido, y escudriñar con vehemencia dónde podemos seguir encontrando bienestar y satisfacción en un planeta lleno de sorpresas, algunas agradables y otras que nos saturan de retos y oportunidades.

\section{REFERENCIAS}

Artiga, S., Garfield, R., \& Orgera, K. (2020). Communities of color at higher risk for health and economic challenges due to COVID-19. Kayser Family Foundation. https://www.kff.org/coronaviruscovid-19/issue-brief/communities-of-color-at-higher-risk-forhealth-and-economic-challenges-due-to-covid-19/

Bao,Y., Sun, Y., Meng, S, Shi, J., \& Lu, L. (2020). 2019-nCoV Epidemic: Address Mental Health Care to Empower Society. Lancet, 395(10224), 37-38. https://doi.org/10.1016/s01406736(20)30309-3

Cevik, M., Bamford, C., \& Ho, A. (2020). COVID-19 pandemic: A focused review for clinicians. Clinical Microbiology and Infection, 20, 842-847. https://doi.org/10.1016/j.cmi.2020.04.023

Connol, K. (2020). Survey reveals gender gap in COVID 19 stress. Psychiatric News. https://doi.org/10.1176/appi.pn.2020.5b3

Crayne, M. (In press). The traumatic impact of job loss and job search in the aftermath of COVID-19. Psychological Trauma.

Cartwright, F., \& Biddiss, M. (2004). Disease \& history. Sutton.

Forbes, M., \& Krueger, M. (2019). The great recession and mental health in the United States. Clinical Psychological Science, 7(5), 114. https://doi.org/10.1177/2167702619859337

Freeman, D., \& Freeman, J. (2013). The stressed sex: Uncovering the truth about men, women, and mental health. Oxford University Press.

Gao, J., Zheng, P., Jia, Y., Mao, Y., Chen, S., Wang, Y., Fu, H., \& Dai, J. (2020). Mental health problems and social media exposure during COVID-19 outbreak. PLoS ONE, 15(4), e0231924. https://doi.org/10.1371/journal.pone.0231924

Girolamo, G., Cerveri, G., Clerici, M., Monzani, E., Spinogatti, \& Vita, A. (2000). Mental health in the coronavirus disease 2019 emergency-The Italian response. JAMA Psychiatry, E2-4. https://doi.org/10.1001/jamapsychiatry.2020.1276

Hooper, M., Nápoles, A. M., \& Pérez, E. (2020). COVID-19 and racial/ethnic disparities. JAMA, 323(24), 2466-2467. https://doi.org/10.1001/jama.2020.8598

Hossain, M. M., Tasnim, S., Sultana, A., Faizah, F., Mazumder, H., Zou, L., McKyer, E. L., J., Ahmed, H. U., \& Ma, P. 2020. Epidemiology of mental health problems in COVID-19: A review. F1000Research, 9, 636. https://doi.org/10.12688/f1000research.24457.1

Huang, Y., \& Zhao, N. (2020). Generalized anxiety disorder, depressive symptoms and sleep quality during COVID-19 outbreak in China: A web based cross-sectional survey. Psychiatry Research, 288, 112954. https://doi.org/10.1016/j.psychres.2020.112954

Jiao, W., Wang, L., Liu, J., Fang, S., Jiao, F., Pettoello-Mantovani,
Financiamiento: La presente investigación no fue financiada por alguna entidad ni patrocinado.

Conflicto de Intereses: No existen conflictos de intereses de parte de los autores de la investigación.

M., \& Somekh, E. (2020). Behavioral and emotional disorders in children during the COVID-19 epidemic. European Pediatric Association, 221, 264-266.e1. https://dx.doi.org/10.1016\%2Fj.jpeds.2020.03.013

Jungmann, S. M., \& Witthoft, M. (2020). Health anxiety, cyberchondria, and coping in the current COVID-19 pandemic: Which factors are related to the coronavirus anxiety? Journal of Anxiety Disorders, $73, \quad 102239$. https://doi.org/10.1016/j.janxdis.2020.102239

Kohhar, R. (2020). Unemployment rose higher in three months of COVID-19 than it did in two years of the Great Recession. FactTank. Pew Research Center.

Lai, M., Ma, S., Wang, Y., Cai, Z., Hu, J., Wei, N. Wu, J., Du, H., Chen, T., Li, R., Tan, H., Kang, S., Wang, Y., Yao, L., Huang, M., Wang, H., Wang, G, Liu, Z., \& Hu, S. (2020). Factors associated with mental health outcomes among health care workers exposed to coronavirus disease 2019. JAMA Newtwork Open.3(3), e203976. https://doi.org/10.1001/jamanetworkopen.2020.3976

Moghanibashi, A. (2020). Assessing the anxiety level of Iranian general population during COVID-19 outbreak. Asian Journal of Psychiatry, 51, 102076. https://doi.org/10.1016/j.ajp.2020.102076

Molesky, M. (2015). This gulf of fire: The great Lisbon earthquake, or apocalypse in the age of science and reason. Vintage.

Nolen-Hoeksema, S. Larson, J., \& Grayson, C. (1999). Explaining the gender differences in depressive symptoms. Journal of Personality and Social Psychology, 77(5), 1061-1072.

NORC at the University of Chicago. (2020). Historic shift in Americans' happiness amid pandemic. University of Chicago.

Ozdin, S., \& Ozdin, S. (2020). Levels and predictors of anxiety, depression and health anxiety during COVID-19 pandemic in Turkish society: The importance of gender. International Journal of Social Psychiatry. https://doi.org/10.1177/0020764020927051

Piquero, A. R., Hawkins, D., \& Kazemian, L. (2012). Criminal carrer patterns. En R. Loeber \& D. P. Farrington (Eds). From juvenile delinquency to adult crime (pp. 14-46). Oxford University Press.

Qiu, J., Shen, B., Zhao, M., Wang, Z., Xie, B., \& Xu, Y. (2020). A nationwide survey of psychological distress among Chinese people in the COVID-19 epidemic. General Psychiatry, 33(2), https://doi.org/10.1136/gpsych-2020-100213

Rossi, R., Socci, V., Pacitti, F., DiLorenzo, G., Marco, A., Siracusano, A., \& Rossi, A. (2020). Mental health outcomes among frontline and second line health care workers during the Coronavirus disease 2019 pandemic in Italy. JAMA Network Open, 3(5), e2010185. https://dx.doi.org/10.1001\%2Fjamanetworkopen.2020.10185 
Pandemias, COVID-19 y Salud Mental: ¿Qué Sabemos Actualmente?

Roy, D., Tripathy, S., Kar, S., Sharma, V., Verma, S., \& Kaushal, V. (2020). Study of knowledge, attitude, anxiety \& perceived mental healthcare need in Indian population during COVID-19 pandemic. Asian Journal of Psychiatry, 51, 102083. https://dx.doi.org/10.1016\%2Fj.ajp.2020.102083

Rumbold, B., \& Dickson, V. (2012). Social determinants of health. En P. Liamputtong, R. Fanany \& G. Verrinder (Eds.), Health, illness and well-being (pp. 177-196). Oxford University Press.

Seo, D., Ahluwaliab, A., Potenzaa, M. \& Sinhaa, R. (2020). Gender differences in neural correlates of stress-induced anxiety. Journal Neuroscience Research, 95(1-2), 15-125. https://doi.org/10.1002/jnr.23926

Spinney, L. (2017). Pale raider: the Spanish flu of 1918 and how it changed the world. Public Affairs.

Tian, F., Li, H., Tian, S., Yang, J., Shao, J., \& Tian, C. (2020). Psychological symptoms of ordinary Chinese citizens based on SCL-90 during level I emergency response to COVID-19. Psychiatry Research, 288, 112992. https://doi.org/10.1016/j.psychres.2020.112992

Tull, M., Edmonds, K., Scamaldo, K., Richmond, J., Rose, J., Gratz, K. (2020). Psychological outcomes associated with stay at home orders and the perceived impact of COVID-19 on daily life. Psychiatry Research, 289, 113098. https://doi.org/10.1016/j.psychres.2020.113098

Vindegaard, N., \& Benros, M. (2020). COVID-19 pandemic and mental health consequences: Systemativ review of the current evidence. Brain, Behavior and Immunity. https://doi.org/10.1016/j.bbi.2020.05.048
Wan, W. (2020). The coronavirus pandemic is pushing America into a mental health crisis. The Washington Post, May 4, p. 12.

Wang, C., Pan, R., Wan, X., Tan, Y., Xu, L., Ho, C. S., \& Ho, R. C. (2020). (2020). Immediate psychological responses and associated factors during the initial stage of the 2019 coronavirus disease epidemic among the general population of China. International Journal of Environmental Research and Public Health, 17(5), 1729. https://doi.org/10.3390/ijerph17051729

Wu, Z., \& McGoogan, J. (2020). Characteristics of and important lessons from the coronavirus disease 2019 outbreak in China. JAMA, 123(13), 1239-1242. https://doi.org/10.1001/jama.2020.2648

Yang, H., \& Ma, J. (2020). How an epidemic outbreak impacts happiness: Factors that worsen (vs. protect) emotional well-being during the coronavirus pandemic. Psychiatry Research, 289, 113045. https://doi.org/10.1016/j.psychres.2020.113045

Zhou, S., Zhang, L., Wang, L. et al. Prevalence and socio-demographic correlates of psychological health problems in Chinese adolescents during the outbreak of COVID-19. European Child Adolescent Psychiatry 29, 749-758. https://doi.org/10.1007/s00787-020-01541-4

Zhu, J., Sun, L., Zhang, L., Wang, H., Fan, A., Yang, B., Li, W., \& Xiao, S. (2020). Prevalence and Influencing Factors of Anxiety and Depression Symptoms in the First-Line Medical Staff Fighting Against COVID-19 in Gansu. Frontiers in psychiatry, 11, 386. https://doi.org/10.3389/fpsyt.2020.00386

\section{Obra bajo licencia de Creative Commons Atribución 4.0 Internacional (CC BY 4.0).}

(C) 2020 Autores. 